The Astrophysical Journal, 243 :L163-L165, 1981 February 1

(c) 1981. The American Astronomical Society. All rights reserved. Printed in U.S.A.

\title{
THE ISOTOPIC COMPOSITION OF SOLAR FLARE ACCELERATED MAGNESIUM
}

\author{
R. A. Mewaldt, J. D. Spalding, E. C. Stone, and R. E. Vogt \\ California Institute of Technology \\ Received 1980 September 8; accepted 1980 October 24
}

\begin{abstract}
We report observations of energetic solar particles from the 1978 September 23 solar flare in which the three stable isotopes of magnesium are clearly resolved with a mass resolution of 0.23 amu. We find ${ }^{25} \mathrm{Mg} /{ }^{24} \mathrm{Mg}=0.14(+0.05,-0.02)$ and ${ }^{26} \mathrm{Mg} /{ }^{24} \mathrm{Mg}=0.15(+0.04,-0.03)$ in the energy interval $12-36 \mathrm{MeV}$ per nucleon. These observations are consistent with terrestrial magnesium isotopic abundances.
\end{abstract}

Subject headings: solar system: general -- Sun: abundances - Sun: flares

\section{INTRODUCTION}

The isotopic composition of the solar system provides key pieces of information for the study of the origin of the elements via nucleosynthetic processes and the study of the formation of the solar system. Although the Sun is the major reservoir of solar system material, most of our present knowledge about the solar system isotopic composition comes from the study of terrestrial material and meteorites, which by weight are trace constituents of the solar system. At present the solar abundances have been studied directly through three channels: spectral analysis of the Sun's emitted light, measurements of the composition of the solar wind, and measurements of the composition of energetic particles accelerated in solar flares. The solar wind and solar energetic particles provide direct samples of material from the solar atmosphere.

In this Letter we report the first observations of solar energetic particles in which the three isotopes of magnesium are clearly resolved.

\section{INSTRUMENT}

The Caltech Heavy Isotope Spectrometer Telescope (HIST) is carried by the ISEE-3 spacecraft, which was launched on 1978 August 12 into a heliocentric orbit approximately 0.01 AU sunward of the Earth. HIST is designed to measure the isotopic composition of solar and galactic cosmic ray nuclei from $\mathrm{Li}$ to $\mathrm{Ni}$ in the energy range from $\sim 5$ to $\sim 250 \mathrm{MeV}$ per nucleon. The HIST is a stack of 11 silicon solid-state detectors. The front two detectors (M1 and M2) are two-dimensional position-sensitive detectors which measure the trajectories of individual particles entering the telescope. Use of this trajectory information results in a significant improvement in the mass resolution compared with telescopes with similar opening angles that do not have trajectory-measuring capability. Following M1 and M2 are nine detectors labeled D1 to D9 from front to back of the detector stack. Further details of the design and operation of HIST, including an instrument cross section, are given in Althouse et al. (1978) and Mewaldt et al. (1979).

\section{OBSERVATIONS}

On 1978 September 23, a large solar particle event was observed by HIST within $1 \mathrm{hr}$ after an importance $2 \mathrm{~B}$ flare located at $35^{\circ} \mathrm{N}, 50^{\circ} \mathrm{W}$ on the Sun. The magnesium data were accumulated from 10:00 UT on 1978 September 23 to 0:00 UT on 1978 September 28, during which more than $10^{4}$ particles with charge $Z \geq 6$ and energy $\geq 5 \mathrm{MeV}$ per nucleon were analyzed. Neon isotope observations from this flare were previously reported by Mewaldt et al. (1979) with the somewhat surprising result (see also Dietrich and Simpson 1979a) that the solar energetic particle composition was consistent with the meteoritic component neon-A which has ${ }^{20} \mathrm{Ne} /{ }^{22} \mathrm{Ne} \approx 8.2$, but not with measurements of the solar wind reported by Geiss (1973), which gave ${ }^{20} \mathrm{Ne} /{ }^{22} \mathrm{Ne} \approx 13.7$.

The method of measuring $\mathrm{Mg}$ isotopes is essentially identical with that employed for our neon measurements. Up to three separate determinations of each particle's mass can be obtained using the individual detector outputs for a multiple $\mathrm{dE} / \mathrm{dx}$ versus residual energy-loss analysis, as described in Mewaldt et al. (1979). Intercomparison of these mass determinations for self-consistency is a powerful technique for background supression. For this analysis, magnesium particles stopping in detectors D2, D3, and D4 (ranges 2, 3 , and 4) were accepted. These ranges include an energy interval of $12-36 \mathrm{MeV}$ per nucleon for ${ }^{24} \mathrm{Mg}$. For each event, a best estimate of the isotope mass $M$ was obtained from a weighted average of the individual mass determinations. For events identified as magnesium, with $M$ between 22 and 28 amu, the consistency check eliminated 52 out of 243 events. The relative isotopic abundances were not sensitive to the criterion used for the consistency check. The histogram for $M$ containing the sum of all accepted events for ranges 2,3 , and 4 is shown in Figure 1. The observed mass resolution obtained by fitting three Gaussian distributions to the histogram is $\sigma=0.23 \mathrm{amu}$.

In Figure 1, there are some events below the ${ }^{24} \mathrm{Mg}$ peak, near ${ }^{23} \mathrm{Mg}$, a radioactive isotope. Instrumental effects such as edge events in the detectors or incom- 


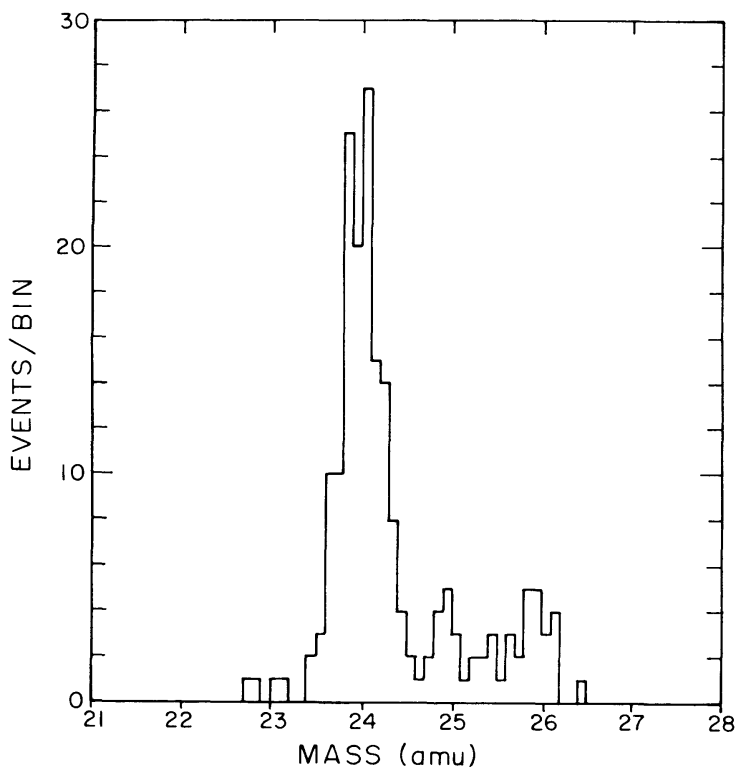

Fig. 1.-Mass histograms for the accepted magnesium events. The energy range for ${ }^{24} \mathrm{Mg}$ is $12-36 \mathrm{MeV}$ per nucleon.

plete charge collection will act to lower the observed mass rather than to increase it. We believe the events near ${ }^{23} \mathrm{Mg}$ to be actual ${ }^{24} \mathrm{Mg}$ events and expect proportionate amounts of background due to ${ }^{25} \mathrm{Mg}$ and ${ }^{26} \mathrm{Mg}$. We have not corrected for this effect because it is much smaller than the statistical uncertainty in our measurements.

The relative isotopic abundances were computed using a two-dimensional maximum likelihood analysis that takes into account the separate mass determinations for each event. Because HIST has a slightly different energy/nucleon acceptance window for each of the magnesium isotopes when events are selected according to range, we made a small correction to the isotopic abundances using the observed magnesium energy spectrum. The correction was $-8 \%$ for the abundance ratio ${ }^{25} \mathrm{Mg} /{ }^{24} \mathrm{Mg}$ and $-14 \%$ for the ratio ${ }^{26} \mathrm{Mg} /{ }^{24} \mathrm{Mg}$. Our $68 \%$ confidence intervals for these ratios are ${ }^{25} \mathrm{Mg} /{ }^{24} \mathrm{Mg}=0.14(+0.05,-0.02)$ and ${ }^{26} \mathrm{Mg} /{ }^{24} \mathrm{Mg}=0.15(+0.04,-0.03)$. These confidence limits take into account only statistical uncertainties, although we believe that the upper limit to systematic uncertainties in these measured ratios is less than 0.01 .

\section{DISCUSSION}

Our solar energetic particle measurement and selected other measurements of magnesium isotopic abundances in the solar system are plotted on a threeisotope diagram in Figure 2 and summarized in Table 1. For a "standard" composition, we take ${ }^{25} \mathrm{Mg} /{ }^{24} \mathrm{Mg}=$ 0.1266 and ${ }^{26} \mathrm{Mg} /{ }^{24} \mathrm{Mg}=0.1398$ from Schramm, Tera, and Wasserburg (1970), based on measurements in a number of terrestrial, lunar, and meteoritic samples.

There are very few direct measurements of magnesium isotopes on the Sun. Boyer, Henoux, and Sotirovski (1971) measured the lines of $\mathrm{MgH}$ above a sunspot and found ${ }^{25} \mathrm{Mg} /{ }^{24} \mathrm{Mg}=0.125 \pm 0.025$ and ${ }^{26} \mathrm{Mg} /{ }^{24} \mathrm{Mg}=0.125 \pm 0.025$ by a curve-of-growth analysis. Lambert, Mallia, and Petford (1971) investigated the $\mathrm{MgH}$ system in the photosphere and in sunspot penumbrae and umbrae, and also measured the isotope shifts of neutral $\mathrm{Mg}$ lines. In sunspot penumbrae they found values ranging from 0.10 to 0.22 for ${ }^{26} \mathrm{Mg} /{ }^{24} \mathrm{Mg}$ and values from 0.11 to 0.19 for ${ }^{25} \mathrm{Mg} /{ }^{24} \mathrm{Mg}$, depending on the $\mathrm{MgH}$ multiplet used in the analysis. The results

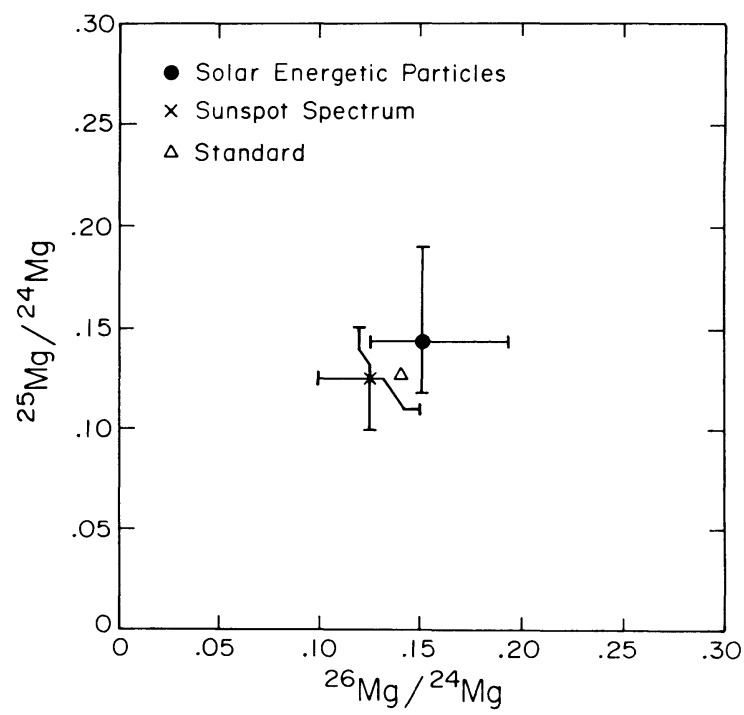

FIG. 2.-A comparison of solar-system magnesium-isotope measurements. The "solar energetic particle" measurement is our own, "sunspot spectrum" is the value reported in Boyer, Henoux, and Sotirovski (1971), and "standard" is from Schramm, Tera, and Wasserburg (1970).

TABLE 1

Solar Energetic Particle Measurement and Other Magnesium Isotopic Abundances

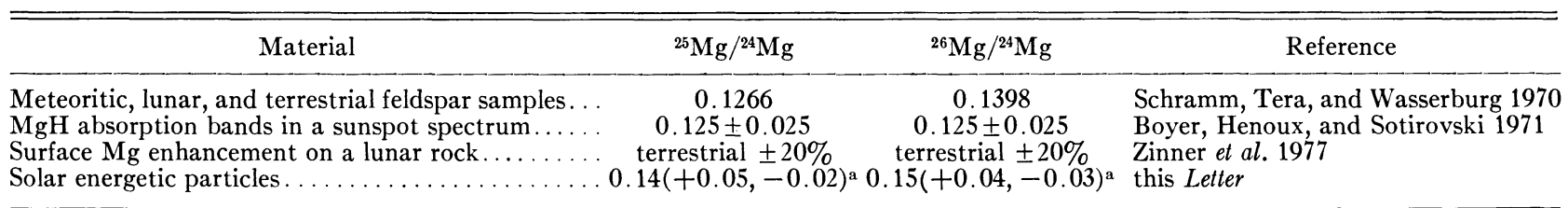

a There is a $16 \%$ probability that the actual ratio is greater and a $16 \%$ probability that it is less than these limits. 
of both of these studies are inconsistent with the significant enhancement in the abundances of the heavier $\mathrm{Mg}$ isotopes reported in earlier work (Branch 1970; Kumar 1969).

The only other measurement of magnesium isotopes in solar energetic particles is a preliminary value reported by Dietrich and Simpson (1979b) based on data summed over seven solar flare events. Although their instrument did not resolve the individual $\mathbf{M g}$ isotopes, they found ${ }^{26} \mathrm{Mg} /{ }^{24} \mathrm{Mg}=0.22 \pm 0.07$, assuming that ${ }^{25} \mathrm{Mg} /{ }^{24} \mathrm{Mg}$ was equal to its terrestrial value.

There are no direct observations of $\mathbf{M g}$ isotopes in the solar wind. Zinner et al. (1977), however, have analyzed surface enhancements of $\mathrm{Mg}$ on lunar rocks that they conclude are due to solar wind implantation. They find magnesium isotopic abundances that are consistent with terrestrial values to within their experimental uncertainties, estimated by Zinner (1979) to be $\sim 20 \%$.

The vast majority of meteoritic samples studied contain "standard" Mg isotope abundances (see, e.g., Schramm, Tera, and Wasserburg 1970). Recently, however, two classes of $\mathrm{Mg}$ isotope anomalies have been discovered in meteorites. Wasserburg, Lee, and Papanastassiou (1977) have observed Mg isotope variations in two inclusions of the Allende meteorite that are characterized by both fractionation (F) effects of several percent, and unknown nuclear (UN) effects of a few tenths of a percent, hence, the designation FUN for this class of anomaly. A second meteoritic abundance anomaly is the result of the in situ decay of radioactive ${ }^{26} \mathrm{Al}$ to ${ }^{26} \mathrm{Mg}$, where ${ }^{26} \mathrm{Mg}$ excesses of up to $\sim 10 \%$ have been reported (Lee, Papanastassiou, and Wasserburg 1977).

Although the magnitude of meteoritic anomalies observed to date is smaller than the present uncertainties in the solar energetic particle observations, the very existence of isotopic anomalies in $\mathrm{Mg}$ and a number of other elements observed in meteorites (see, e.g., the reviews by Clayton 1978; Podosek 1978) demonstrates that the solar system is not homogeneous in its isotopic composition and thereby emphasizes the importance of an accurate determination of the solar composition. Within the current statistical limitations, we find no evidence that the composition of the 1978 September 23 solar flare is different from the terrestrial magnesium standard.

W. E. Althouse, A. C. Cummings, and T. L. Garrard made significant contributions to the design and development of the HIST instrument. This work was supported in part by NASA under contract NAS5-20721 and grant NGR 05-002-160.

\section{REFERENCES}

Althouse, W. E., Cummings, A. C., Garrard, T. L., Mewaldt, R. A., Stone, E. C., and Vogt, R. E. 1978, Geosci. Electron., 16, 204.

Boyer, R., Henoux, J. C., and Sotirovski, P. 1971, Solar Phys., 19,330 .

Branch, D. 1970, A p. J., 159, 39.

Clayton, R. N. 1978, Ann. Rev. Nucl. Part. Sci., 28, 501.

Dietrich, W. F., and Simpson, J. A. 1979a, Ap. J. (Letters), 231, L91.

L. 1979b, Proc. 16th Internat. Cosmic Ray Conf., 5, 85.

Geiss, J. 1973, 13th Internat. Cosmic Ray Conf. (Denver: University of Denver), 5,3375 .

Kumar, C. K. 1969, Bull. AAS, 1, 351.
Lambert, D. L., Mallia, E. A., and Petford, A. D. 1971, M.N.R.A.S., 154, 265 .

Lee, T., Papanastassiou, D. A., and Wasserburg, G. J. 1977, A p. J. (Letters), 211, L107.

Mewaldt, R. A., Spalding, J. D., Stone, E. C., and Vogt, R. E. 1979, A p. J. (Letters), 231 , L97.

Podosek, F. A. 1978, Ann. Rev. Astr. Ap., 16, 293.

Schramm, D. N., Tera, F., and Wasserburg, G. J. 1970, Earth Planet. Sci. Letters, 10, 44.

Wasserburg, G. J., Lee, T., and Papanastassiou, D. A. 1977, Geophys. Res. Letters, 4, 299.

Zinner, E. 1979, private communication.

Zinner, E., Walker, R. M., Chaumont, J., and Dran, J. C. 1977, Proc. 8th Lunar Sci. Conf., 3, 3859.

Richard A. Mewaldt, John D. Spalding, Edward C. Stone, and Rochus E. Vogt: California Institute of Technology, Mail Code 220-47, Pasadena, CA 91125 